\title{
Implementing Teams in a Patient-Centered Medical Home Residency Practice: Lessons Learned
}

\author{
Tsveti Markova, MD, Maribeth Mateo, MD, and Linda M. Roth, PhD
}

Introduction: The "new model of care" calls for a new approach for primary care delivery that focuses on patient centeredness, quality, safety, effective and efficient care, and interdisciplinary teamwork. Medical education needs to parallel this health care reorganization. Implementing a team approach in a residency practice, especially in ambulatory settings, poses unique challenges.

Methods: We introduced interdisciplinary teams in a family medicine residency site, integrating clinical and educational objectives.

Results: We report our challenges and successes in the transformational journey to a patient-centered medical home, for which a team approach is critical to achieving high quality care.

Conclusion: Establishing high-functioning interdisciplinary teams takes leadership commitment; the engagement of everyone in the practice; investment in staff, resident, and faculty development; and clear communication of vision and goals. Integration of clinical and educational objectives can be powerfully synergistic. Clinical, organizational, and educational outcomes are needed to evaluate impact. (J Am Board Fam Med 2012;25:224-231.)

Keywords: Family Medicine, Health Care Reform, Health Care Team, Medical Home, Patient-Centered Care

Over the last decade there has been a call for change in the US health care system. Several reports by the Institute of Medicine, including To Err is Human-Building a Safer Health Care System ${ }^{1}$ and Crossing the Quality Chasm, ${ }^{2}$ have highlighted the critical need for developing a new approach to patient care that focuses on patient safety and the delivery of high-quality care and requires new physician competencies that include working in interdisciplinary teams, which in turn necessitates team training for all current and future physicians. ${ }^{3,4}$ In concert with changes in approaches to clinical care, medical education curricula must be updated to parallel the changes in the health care delivery system. ${ }^{5,6}$ Although the health care literature provides some direction for how to create and maintain

This article was externally peer reviewed.

Submitted 23 May 2011; revised 3 November 2011; accepted 4 November 2011.

From the Department of Family Medicine and Public Health Sciences, Wayne State University School of Medicine, Rochester Hills, MI.

Funding: none.

Conflict of interest: none declared.

Corresponding author: Tsveti Markova, MD, $1101 \mathrm{~W}$. University Drive, 3 North, Rochester Hills, MI 48307 (E-mail: tmarkova@med.wayne.edu). high-functioning teams in large organizations and inpatient settings ${ }^{7-10}$ - in which interdisciplinary health care teams have become an expectationsuch approaches are difficult to implement in outpatient settings. Doing so is even more difficult when ambulatory residency education is a mission of the practice.

Family medicine residency educators are charged with developing team-based care competencies in their learners. ${ }^{11,12}$ The recent national focus on the patient-centered medical home (PCMH) emphasizes the importance of these competencies. In fact, 6 of the 14 programs participating in the Preparing the Personal Physician for Practice $\left(\mathrm{P}^{4}\right)$ project, a 6-year national comparative case study of innovations associated with the PCMH in family medicine residency training, chose team-based care and training in teams as their focus area. ${ }^{13}$ The PCMH is a "patient-centered, multifaceted source of personal primary health care...based on a relationship between the patient and physician, formed to improve the patient's health across a continuum of services." 14 Four "pillars" make up the PCMH: practice organization, health information technology, quality measures, and patient experience. ${ }^{15}$ As 
a component of practice organization, teamwork is essential to the PCMH.

Transformation to a PCMH requires not only implementing new, sophisticated office systems; a practice must also adopt substantially different approaches to patient care. Such a fundamental shift nearly always challenges doctors to re-examine their identities as physicians. For example, transformation involves a move from physician-centered care to a team approach in which care is shared among adequately prepared office staff. ${ }^{16}$ Our previous work demonstrated that the implementation of interdisciplinary teams improved learning opportunities and quality of teaching, job satisfaction, employee autonomy, staff roles, and staff attitudes toward residents. ${ }^{17}$ Therefore, when we established a new residency program in 2007, we introduced a team approach during our journey toward designation as a PCMH. Our aim was to maintain an organizational climate that facilitates continuous improvement in a high-quality clinical and educational environment. A team approach was essential to achieving this goal.

Teams consist of 2 or more individuals with complementary skills who have a common commitment and purpose and share a set of performance goals for which they hold themselves mutually accountable. ${ }^{4}$ Primary care teams have been defined by others as "a group with a specific task or tasks, the accomplishment of which requires the interdependent and collaborative efforts of its members." ${ }^{16}$ We further defined our health care teams as groups of individuals functioning at the same time and in the same place to accomplish common goals in a collaborative fashion. ${ }^{17}$

\section{Methods: Our Experience \\ Setting}

Built in 2008, the Wayne State University Family Medicine Center (FMC) in Rochester, Michigan, is a state-of-the art facility designed to function specifically in the "new model of care" and support PCMH principles. Informed by the Future of Family Medicine report, ${ }^{11}$ the "new model of care" proposes transformational redesign of both work and workplaces to better serve the changing needs of patients, physicians, and practice teams. Adjacent to a large, community-based medical center that provides a full continuum of clinical programs, the FMC comprises 13,600 square feet of space and 28 examination rooms, 23 of which are patient examination rooms; 2 large pediatrics room that can accommodate a whole family; a counseling room; and 2 procedure rooms. It is home to 6 physician faculty members, a behavioral health psychologist, 2 psychology interns, 20 family medicine residents, a clinical manager, 8 nursing staff (of whom two are Licensed Practical Nurses [LPNs]), and a billing and coding coordinator who is responsible for care management. The rest of the nursing staff consists of medical assistants (MAs), 3 of whom work primarily in the front office (checking patients in and out); 2 function in the back of the office, and one floats. The 6 faculty physicians have various teaching responsibilities, including inpatient adult medicine and obstetric rounds, precepting in the FMC, developing curriculum and didactic presentations, counseling residents, and mentoring residents in research and scholarship. In addition, their clinical responsibilities include 1 to 4 half-day sessions per week, during which they provide direct patient care in the FMC, totaling 2 clinical full-time equivalent with an average productivity of 8.3 relative value units per 3.5-hour session. The FMC medical director has $10 \%$ of his time designated for administrative management of clinical operations. In 2010, during its second year of operations, the FMC's total relative value units (including all residents and faculty visits) was 12,600 , with a $20 \%$ increase in patient visits and a $32 \%$ increase in patient revenue from the previous year. The office operates in a positive financial balance. Our practice has earned $\mathrm{PCMH}$ designation by Blue Cross/Blue Shield of Michigan, as well as a commendation by the Accreditation Council of Graduate Medical Education for being a national model for implementing $\mathrm{PCMH}$ principles in residency education. We are currently applying for national recognition through URAC's Patient Centered Health Care Home Practice Achievement Program.

\section{Teams in the PCMH}

As the main ambulatory training site for family medicine residents, the FMC was structurally designed to operate with 3 interdisciplinary health care delivery teams, each with its own geographic identity. The team space was clearly identified with a team nurses' station surrounded by 8 patient examination rooms. Because the team concept was implemented during the second year of operations, 
the FMC was not functioning at its full capacity; therefore we decided to create 2 health care delivery teams using only two thirds of the office space (16 examination rooms). At the present time, each team comprises a front desk practice greeter, an LPN, an MA, 10 residents, 3 faculty members, and a floating psychology intern. The clinical teams practice independently, but there is cross-coverage by the MA floater in the case of emergencies. The front desk staff is assigned to a specific team for certain functions, such as responding to patient messages and authorizing referrals. However, they cross-cover each other for billing and payment collections to assure uniform adherence to the appropriate FMC policy as well as to streamline the checkout process. In addition to the 2 core teams, we utilize the Community Care Travel Team (CCTT) as an extension of our practice. It is organized by our local physician organization and visits different physicians' offices on a monthly basis. The CCTT includes a nurse educator, a dietician, an exercise physiologist, and a wellness coach. We refer patients with chronic illnesses to the CCTT, and our staff and residents participate in monthly visits that are scheduled in the FMC for better continuity of care. Each patient is scheduled (in half-hour increments) for a face-to-face visit with the CCTT health care providers who meet their needs, joined by a resident or our FMC staff.

Elements required for building effective and sustainable teams include developing team leadership, revising job descriptions, cross-training staff, communicating clear goals, setting measurable objectives, and publically praising teams and individuals for accomplished tasks. The goal is to create and maintain an environment in which everyone feels both respected and empowered to actively contribute to the continuous improvement of our clinic's processes.

Our strategy for the structure of team membership has evolved. At the beginning of the academic year we randomly assigned residents and faculty to the 2 health care teams. Faculty share responsibilities as physician team leaders because they are not full time in the FMC. They also became the academic advisors and mentors for the team's residents. The office manager then selected nursing staff with similar personalities or work styles to work with each team. For several months after that, because of personality conflicts within the teams, we made occasional reassignments of staff, hoping for a better fit. This approach was found to be unsuccessful and we stopped moving staff between teams. Our current teams are composed of individuals with complementary skill sets who are now coached to embrace the differences among their team members.

We discovered that we needed another structural adjustment, this time related to team leadership. We initially hired LPNs as team leaders, but we discovered quickly that during the day-to-day operations an MA often naturally took on the leadership role. Team leadership is now shared between a faculty physician, a resident, and a nursing staff member who is either an LPN or an MA, depending on the leadership characteristics and behaviors demonstrated across nursing staff members of each team. The team faculty who is precepting for the particular session assumes the team leader responsibilities and supervises day-to-day operations and, with the resident leader, facilitates regular team meetings and monitors quality management activities. Nursing team leaders are involved in direct patient care and have administrative duties as well. Their direct patient care activities include review of patient charts, management of chronic diseases (eg, initiating standing orders for diabetic patients or providing specific patient education), identification of gaps in care, and leadership of team huddles. Nursing staff members' administrative responsibilities include management of immunizations and maintenance of our electronic patient registry. Most of their administrative duties are incorporated into their daily schedules, but occasionally the clinical manager assigns them protected time for the completion of bigger projects. Additional general office duties are further distributed within each team and include scheduling, answering the phone (taking messages and prescription refill requests), reviewing e-charts for the next day, verifying insurance, supervising daily patient flow, accommodating walk-ins, checking out patients, and stocking rooms. Each team is also assigned specific "all-clinic" responsibilities such as equipment maintenance and procurement of supplies. We redesigned individual team member job descriptions to set performance expectations for each member. For example, given that we are a paperless office, our MAs are required to demonstrate the ability to utilize electronic health records (EHRs). This helps them to visualize their role and how it relates to the overall patient care experience 
and practice flow. With increased understanding of the different members' responsibilities, there is an overall appreciation for all elements of the patient care experience.

\section{Effective Communication}

In The Five Dysfunctions of a Team, Lencioni ${ }^{18}$ notes that members of effective teams trust one another, engage in unfiltered conflict around ideas, commit to decisions and plans of action, hold one another accountable for delivering against those plans, and focus on achieving collective results. The second dysfunction Lencioni describes focuses on the importance of mastering conflict. This requires open and honest communication among team members, allowing them to voice their opinions even at the risk of causing disagreement. ${ }^{18}$ Specifically for health care teams, Grumbach and Bodenheimer ${ }^{16}$ describe effective communication as a key characteristic of a cohesive team. Carving out time for routine communication among the members of each team and among the entire practice group is essential. We incorporated several structured processes for this opportunity to occur.

\section{Daily Team Huddles}

Each half-day session begins (at 8:15 AM or at 1:15 PM) with a structured huddle, which is a practice tool to help the efficiency of patient flow. The lead nursing staff member facilitates the huddle, during which the team discusses logistics such as the num- ber of patients scheduled for each physician and the unanticipated absence or delay of any team members. In addition, the team leader may reinforce new practice initiatives relevant to the care of the patient population. For example, with a focus on the management of diabetes, team leaders will verbally remind the team of the "self-management goal" section in the diabetic template in our EHR, NextGen (NextGen Healthcare, Horsham, PA). To ensure consistency in the huddle process, we developed a checklist designed for daily use, as indicated in Figure 1. The checklist is also reviewed by nursing staff that start later on a particular day so they stay abreast of the huddle discussions and updates.

\section{Team Meetings}

Team meetings are scheduled quarterly during the noon hour, when all team members come together to discuss current issues. All residents, faculty, and staff are required to attend and expected to arrange for their own lunch. Staff take a lunch break before or after the meeting. Topics include patient care issues such as identified gaps within the team's patient population as well as team productivity. The team then designs improvement projects and Plan Do Study Act (PDSA) cycles to improve care (see Figure 2). The management team encourages utilization of PDSAs as an important component of the model for continuous improvement. This provides a mechanism to think in a constructive way to

Figure 1. Huddle checklist.

\author{
Huddle Checklist \\ $\square$ Review current patient appointment schedule with faculty/resident and identify openings. \\ $\square$ Discuss the weekly report of the average time the patient spends with each member of the \\ team (medical assistants, resident, preceptor). \\ $\square$ Discuss any procedures scheduled and equipment required. \\ $\square$ Discuss phone duty and preceptor assignments for the day. \\ $\square$ Give forms for review or signature to faculty or residents at each huddle session. \\ $\square$ Identify patients with diabetes, asthma, hypertension or other chronic illness for disease \\ management issues and referral to Chronic Care Travel Team. \\ Identify obstetric patients. \\ Discuss outstanding issues or concerns from previous day's clinic sessions. \\ Identify additional faculty available for precepting. \\ Identify each preceptor available for each station. \\ Review nurse visits with preceptor.
}


Figure 2. Plan Do Study Act (PDSA) cycle worksheet.

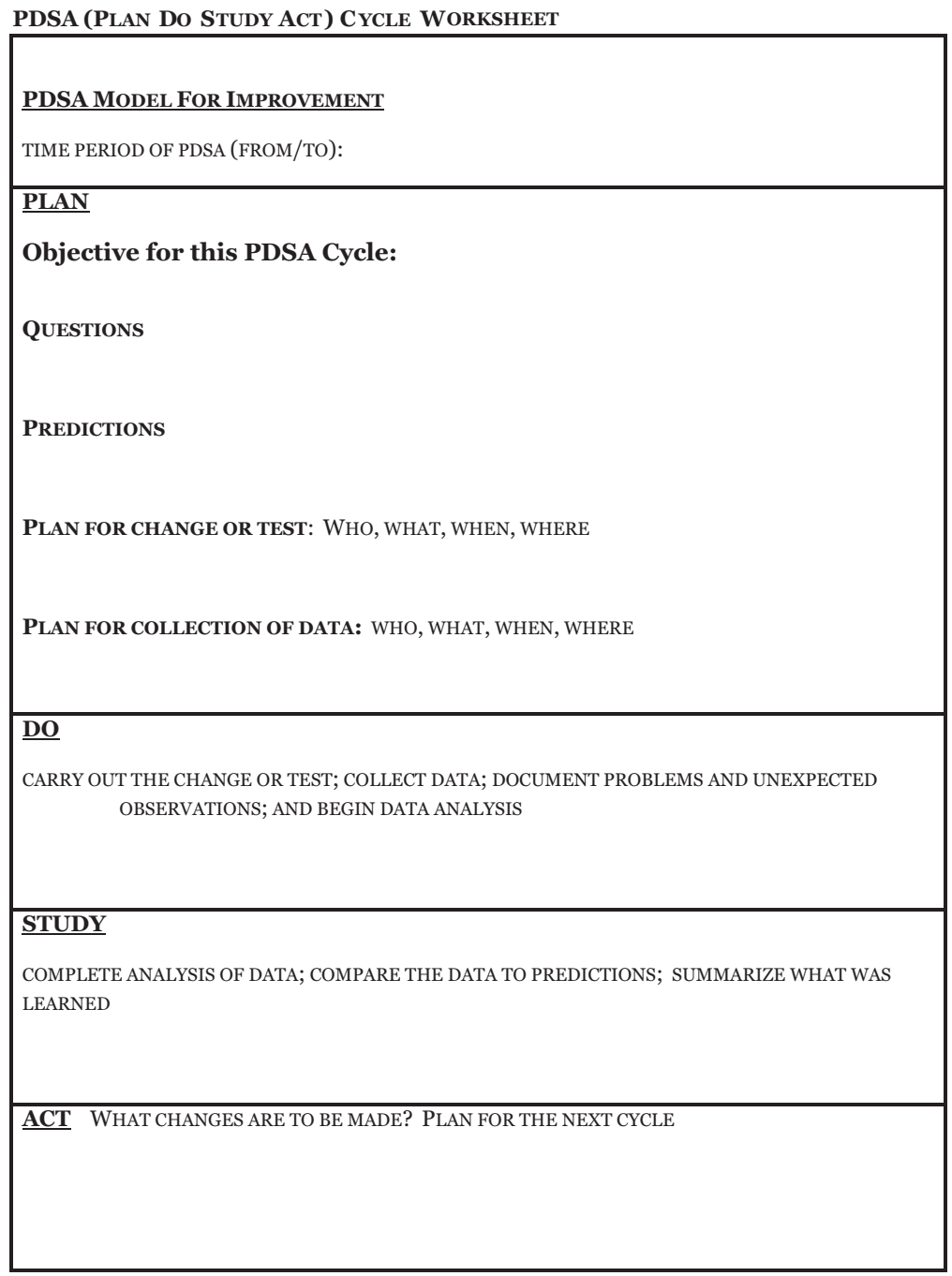

identify a problem, design a short-term plan for a small-scale intervention, and collect and analyze results. If the intervention is successful, then it is implemented practice wide. Designing PDSAs as a team empowers all members to bring their unique contribution in the problem-solving activity. Team meetings reinforce the importance of the team approach and help members to stay engaged with each other.

\section{Clinical Operations Meetings}

We commit 1 hour per month to a practice-wide clinical operations meeting that is devoted to collaborative learning among the entire practice team. Led by the medical director and office manager, the agenda may include EHR enhancements, reports of patient satisfaction surveys, and new or revised pol- icies or procedures. It also is a time for the individual teams to present their projects and initiatives, both successful and failed, to the entire practice. Team sharing is both an informative and developmental process through which we continue to highlight $\mathrm{PCMH}$ principles. It also allows a venue for sharing successful initiatives to be considered for practice-wide adoption.

\section{Practice Improvement Teams}

Our practice improvement (PI) teams consistently review our processes and procedures. During a rotation each month, a group of 3 to 4 residents join some of our faculty and staff who are conducting a PI project. As an example, our practice recently elected to focus on diabetes mellitus as an area of concentration and we developed a patient registry. 
The PI team updates the patient registry on a monthly basis and identifies gaps in care. They develop a PDSA cycle to address these gaps. At the close of each month, residents present their outcomes at our clinical operations meeting. The following month, the next PI team builds on the knowledge gained from the previous cycle and develops a new PDSA cycle. Although a single cycle may provide a great deal of information, it may take several cycles before a measurable outcome is achieved. For example, the goal of our current diabetic PI project is to improve care by helping diabetic patients to control their disease. This can be objectively measured by following $\mathrm{HgA1C}$ levels. Figure 3 demonstrates how a series of PDSA cycles was necessary for notable change in $\mathrm{HgAlC}$ measurements.

When a change leads to improvement, teams throughout the practice adopt the new process, and the clinical manager and medical director monitor the change to anchor it into our practice. Because they are engaged in the process of improvement, residents are empowered to lead the team to test changes as they see gaps in care. With this process, resident leaders have emerged, and now we have 5 residents ( 2 in postgraduate year 1, 2 in postgraduate year 2 , and 1 in postgraduate year 3 ) as $\mathrm{PCMH}$ Resident Champions; they are an invaluable re-

Figure 3. Serial Plan Do Study Act (PDSA) cycles impacting glycosylated hemoglobin (HbA1c) outcomes. EMR, electronic medical record.

$\underline{\text { Serial PDSA Cycles, Impacting HgA1C Outcomes }}$

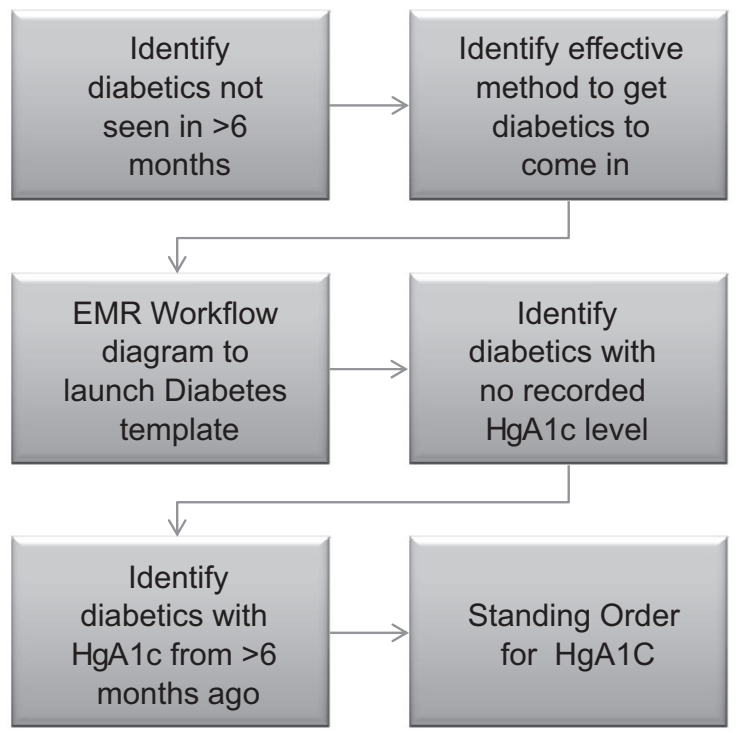

source for other residents in designing their quality improvement projects and discussing FMC issues.

\section{Didactic Presentations About the Chronic Care Model and PCMH Principles}

We have discovered that we are all learners in the process of $\mathrm{PCMH}$ practice transformation and that faculty, residents, and staff need to learn together. Therefore, we present to the entire practice didactics about topics related to the PCMH model. Each presentation includes a theoretical background as well as practical ideas for implementation into our daily practice. These presentations have proven to be quite beneficial in the development of all of our team members. Some relevant topics have included accountable care organizations, meaningful use of the EHR, patient self-management, and transitions in care between different health care facilities.

\section{Engagement in a Learning Collaborative}

We also enrolled the FMC in the Mackinac Learning Collaborative, ${ }^{19}$ which includes 14 southeast Michigan practices in a process of registry implementation and PCMH transformation. The collaborative provides infrastructure for monthly reporting of clinical parameters and coaching resources for individual practices. Monthly phone conferences and quarterly Mackinac Learning Collaborative learning sessions provide an opportunity for representative staff, team leaders, residents, and faculty to share our best practices and learn from other organizations in the transformation process. These learning sessions help maintain and rejuvenate the engagement of the teams in our own practice and help reduce change fatigue among the team members. Attendees bring the information learned back to the practice to generate new ideas for improved processes.

\section{Discussion of Lessons Learned and Future Steps}

Implementation of team-based care was essential for our PCMH transformation. It required a practice culture change to a shared leadership model that began with the physicians and included the nursing staff and other health care providers. The physician leaders in each team modeled the paradigm change from an authoritative role to facilitative leadership. Nursing team leaders were given a great deal of responsibility outside routine patient 
care and were expected to make decisions independently. We have learned that this shift in culture is achievable over time but requires continuous monitoring, education, support, and development. We are currently collecting preliminary data related to team members' perspectives on change in our clinic's organizational culture and their acquisition of PCMH knowledge.

We also have found that the most effective individuals to lead teams are not necessarily those with the most advanced degrees or even the most clinical experience. For example, initially we focused on recruiting 2 LPNs as team leaders, assuming that their more extensive training, compared with that of MAs, would have better prepared them for a leadership role. Despite extensive mentoring by the office manager and medical director, neither LPN team leader successfully fulfilled the expectations provided in their job description, as was evident in their semi-annual performance evaluations. Both LPNs were eventually replaced by MAs from their respective teams, who embraced the principles of the PCMH transformation and enthusiastically led their teams' PI initiatives.

Effective communication among team members is critical, but it is challenging to achieve. Although time is set aside daily during team huddles and monthly and quarterly during clinical operations and team meetings, respectively, it is still difficult to maintain everyone's engagement. To accomplish and sustain organizational culture change, all team members need to be empowered to speak freely and initiate a process of improvement. Faculty physicians are physician leaders, but they also are learners, and sometimes they struggle with the concept of teaching the $\mathrm{PCMH}$ principles because they are still "under development." We realized that the $\mathrm{PCMH}$ designation process and participation in a learning collaborative can provide a framework for both clinical and educational transformation. Consistent with the findings from the National Demonstration Project, ${ }^{20}$ we discovered that the transformation of a practice into a PCMH is not a simple task. It takes a redesign of the entire practice in a continuous process for becoming a learning organization.

To evaluate progress we need a robust array of evaluation tools. We currently are collecting data on several educational, organizational, and clinical outcome measures. As more practices participate in this process and share information, dissemination of their collective outcomes data will provide invaluable guidance to others.

Medical education curricula are evolving as we integrate more clinical principles into our practices. Integrating the clinical transformation to PCMH and experiential teaching about it is powerfully synergistic. As medical students rotate in the FMC for their family medicine clerkship, they also are exposed to this model of practice. The new medical education principles need to be incorporated in the whole continuum of medical education: from medical students and residents to practicing physicians and faculty. ${ }^{5}$

\section{Conclusion}

Our aim is to maintain an organizational climate that facilitates continuous process improvement in a high-quality clinical and educational environment. We have made great strides in our PCMH transformation and our implementation of teambased care and education, yet it continues to be a work in progress because there is no defined road map for our journey. We hope that we can contribute to defining that road map.

\section{References}

1. Kohn LT, Corrigan JM, Donaldson MS, eds, Committee on Quality of Health Care in America. To err is human: building safer health system. Washington, DC: National Academy Press; 2000.

2. Committee on Quality Health Care in America. Crossing the quality chasm: a new health system for the 21 st century. Landover, MD: Institute of Medicine; 2001.

3. Greiner AC, Knebel E, eds. Health professions education: a bridge to quality. Quality Chasm Series. Washington, DC: National Academies Press; 2001:175.

4. Morrison G, Goldfarb S, Lanken P. Team training of medical students in the 21st century: would Flexner approve? Acad Med 2010;85:254-9.

5. Irby DM, Cooke M, O'Brien BC. Calls for reform of medical education by the Carnegie Foundation for the Advancement of Teaching: 1910 and 2010. Acad Med 2010;85:220-7.

6. Katzenbach J, Smith D. The discipline of teams. Harv Bus Rev 2005;83:162-71.

7. Dunn EJ, Mills PD, Neily J, Crittenden MD, Carmack AL, Bagian JP. Medical team training: applying crew resource management in the Veterans Health Administration. Jt Comm J Qual Patient Saf 2007;33:317-25.

8. Flin R, Maran N. Identifying and training non-technical skills for teams in acute medicine. Qual Saf Health Care 2004;13(Suppl 1):i80-84. 
9. Salas E, Wilson KA, Murphy CE, King H, Salisbury M. Communicating, coordinating, and cooperating when lives depend on it: tips for teamwork. Jt Comm J Qual Patient Saf 2008;34:333-41.

10. Wilson KA, Burke CS, Priest HA, Salas E. Promoting health care safety through training high reliability teams. Qual Saf Health Care 2005;14:303-9.

11. Martin JC, Avant RF, Bowman MA, et al. The future of family medicine: a collaborative project of the family medicine community. Ann Fam Med 2004; 2(Suppl 1):S3-32.

12. Pugno PA, ed. Residency program solutions: criteria for excellence. 8th ed. Leawood, Kansas, AAFP; 2011.

13. Carney PA, Eiff MP, Green LA, et al. Preparing the personal physician for practice $\left(\mathrm{P}^{4}\right)$ : site-specific innovations, hypotheses, and measures at baseline. Fam Med 2011;43:464-71.

14. Rosenthal TC. The medical home: growing evidence to support a new approach to primary care. J Am Board Fam Med 2008;21:427-40.

15. American Academy of Family Physicians. Patientcentered medical home checklist. Available at http:// www.aafp.org/online/etc/medialib/aafp_org/documents/ membership/pcmh/checklist.Par.0001.File.tmp/PCMH Checklist.pdf. Accessed May 1, 2011.

16. Grumbach K, Bodenheimer T. Can health care teams improve primary care practice? JAMA 2004; 291:1246-51.

17. Roth LM, Markova T, Monsur JC, Severson RK. Effects of implementation of a team model on physician and staff perceptions of a clinic's organizational and learning environments. Fam Med 2009; 41:434-9.

18. Lencioni P. The five dysfunctions of teams: a leadership fable. San Franscisco, CA; Jossey-Bass; 2002.

19. Practice Transformation Institute. Mackinac Learning Collaborative. Available at http://www.transformcoach. org/EducationCME/MackinacLearningCollaborative. aspx. Accessed May 1, 2011.

20. Nutting PA, Miller WL, Crabtree BF, Jaen CR, Stewart EE, Stange KC. Initial lessons from the first national demonstration project on practice transformation to a patient-centered medical home. Ann Fam Med 2009:7:254-60. 